\title{
Editorial
}

\section{Non-traditional risk factors for obesity and overweight in children: First insights on Georgia}

\author{
Dario Gregori $^{\mathrm{a}, *}$, Ivane Chkhaidze ${ }^{\mathrm{b}}$ and Lorenza Caregaro Negrin ${ }^{\mathrm{c}}$ \\ ${ }^{a}$ Unit of Biostatistics, Epidemiology and Public Health, Department of Cardiac, Thoracic and Vascular Sciences, \\ University of Padova, Italy \\ ${ }^{\mathrm{b}}$ M. Iashvili Central Children Hospital, Tbilisi State Medical University, Tbilisi, Georgia \\ ${ }^{\mathrm{c}}$ Department of Medicine, University of Padova, Italy
}

Received 2 May 2017

Accepted 18 May 2017

After the breakdown of the Soviet Union, we have been witnessing the creation of novel, independent, nations that, while keeping more or less tight relationships with Russia, positioned themselves as emerging markets and growing economies. However, studies on the impact of such transition are lacking, given the poor availability of quality data and temporal trends [1], which are available mainly for Eastern Europe countries, more than on Eurasian or Transcaucasia regions. Georgia, in particular, is one of the countries where the closeness to the Western world has been more pronounced. Indeed, the steady economic growth, which has occurred over the past decade, has increased the accessibility of children to most of the factors which are known to be potentially related with obesity and overweight in kids living in Western countries. This reflects in an increased obesity rate, which is ranging between $20 \%$ and $30 \%$ in children, with a positive trend [2]. However, only a few data and research are available on Georgia for what concerns such aspects. A seminal work of Kherkheulidze and colleagues [3] has indicated an insufficient knowledge among students and parents about nutrition and nutrition-related factors.

The aim of this collection of research works is twofold: (i) to provide the Georgian public health community with preliminary data on the impact of non-traditional risk factors on nutrition and overweight, and (ii) to make available some methodological approaches useful in stimulating new researchers and in getting new evidences.

The three papers included in this collection present different perspectives on the effect that novel risk factors may play on Georgian children's overweight/obesity risk. The paper from Lorenzoni et al. provides evidence, from an experimental study, on the impact on energy intake of television watching and advertising. The other paper from the same author puts the Georgian epidemiology in a broader context, comparing distribution of risk

\footnotetext{
*Corresponding author: Dario Gregori, Unit of Biostatistics, Epidemiology and Public Health, Department of Cardiac, Thoracic and Vascular Sciences, University of Padova, Via Leonardo Loredan 18, 35131 Padova, Italy. Tel.: +39 049 8275384; Fax: +39 02 700445089; E-mail: dario.gregori@unipd.it.
} 
factors and their consequences on children obesity with other countries. Finally, the paper from Vecchio et al. describes the Georgian cultural and linguistic adaptation of an international instrument, the IBAI (International Brand Awareness Instrument), which is aimed at measuring the effect that advertising has on children.

We hope that the availability of such initial research will contribute to open the door for future investigations on obesity in children and their determinants in Georgia and in the region.

\section{References}

[1] Stillman S. Health and nutrition in Eastern Europe and the former Soviet Union during the decade of transition: A review of the literature. Economics \& Human Biology. 2006;4(1):104-46.

[2] Ng M, Fleming T, Robinson M, Thomson B, Graetz N, Margono C, et al. Global, regional, and national prevalence of overweight and obesity in children and adults during 1980-2013: A systematic analysis for the Global Burden of Disease Study 2013. The Lancet. 2014;384(9945):766-81.

[3] Kherkheulidze M, Kavlashvili N, Kandelaki E, Manjavidze T. Evaluation of nutritional knowledge of second grade school children and assessment of their dietary intake. Georgian Med News. 2012;212:58-64. 